\title{
On the Dimension of Bivariate Superspline Spaces
}

\author{
By Charles K. Chui and Tian Xiao He*
}

\begin{abstract}
A bivariate piecewise polynomial function of total degree $d$ on some grid partition $\Delta$ that has $r$ th order continuous partial derivatives everywhere may have higher-order partial derivatives at the vertices of the grid partition. In finite element considerations and in the construction of vertex splines, it happens that only those functions with continuous partial derivatives of order higher than $r$ at the vertices are needed to give the same full approximation order as the entire space of piecewise polynomials. This is certainly the case for $d \geq 4 r+1$. Such piecewise polynomial functions are called supersplines. This paper is devoted to the study of the dimension of certain superspline spaces. Since an exact dimension would have to depend on the geometric structure of the partition $\Delta$, we will give only upper and lower bounds. We will show, however, that the lower bound value is sharp for all quasi-crosscut partitions; and under suitable assumptions on $r$ and $d$, the upper and lower bounds agree on both type-1 and type-2 arbitrary triangulations. In addition, a dimension criterion which guarantees that the lower bound gives the actual dimension is given.
\end{abstract}

1. Introduction. Let $\Omega$ be a closed simply connected polygonal region in $\mathbf{R}^{2}$ and $\Delta$ a partition on $\Omega$ consisting of straight line segments, called edges. Then $\Delta$ is called a rectilinear grid partition of $\Omega$, and the points of intersection of the edges are called the vertices (or grid points) of $\Delta$. The (closed) polygons which are the closures of the components of the complement of $\Delta$ relative to $\Omega$ are called the components of the partition. For any two nonnegative integers $r$ and $d$, the vector space $S_{d}^{r}=S_{d}^{r}(\Delta)=S_{d}^{r}(\Delta, \Omega)$ of functions $f \in C^{r}(\Omega)$ such that the restrictions of $f$ to each component of the grid partition are in $\pi_{d}$, the space polynomials in two variables of total degree not exceeding $d$, is called a bivariate spline space. In this paper, we are interested in the study of the dimension of the subspace $\hat{S}_{d}^{r}=\hat{S}_{d}^{r}(\Delta)=\hat{S}_{d}^{r}(\Delta, \Omega)$ of functions $f$ in $S_{d}^{r}$ which have all partial derivatives of order at least $2 r$ relative to $\Omega$ at each (interior or boundary) vertex of the partition. The subspace $\hat{S}_{d}^{r}$ is called a superspline space in [2]. It is well known that when $\Delta$ is a (regular) triangulation, the approximation order of $\hat{S}_{d}^{r}$ is the same as that of the full space $S_{d}^{r}$, namely $d+1$, provided that $d \geq 4 r+1$ (cf. [2], [9], [10]), and in fact bivariate supersplines with minimal supports in $\hat{S}_{d}^{r}$ (called vertex splines) are shown to form a basis of $\hat{S}_{d}^{r}$ and can be constructed explicitly (cf. [2]).

It is also well known that in the study of $\operatorname{dim} S_{d}^{r}$, the dimension of the full spline space $S_{d}^{r}$, it is in general not possible to give an exact formula for $\operatorname{dim} S_{d}^{r}$

Received May 23, 1988.

1980 Mathematics Subject Classification (1985 Revision). Primary 41A15, 41A25, 41A63.

Key words and phrases. Bivariate splines, dimension, supersplines, lower and upper bound, quasi-crosscut partition, type-1 and type-2 triangulations, dimension criterion.

* Research supported by SDIO/IST managed by the U.S. Army Research Office under Contract number DAAL03-87-K-0025 and the National Science Foundation under grant numbers DMS-8602337 and DMS-87-01190. 
in terms of the number of vertices, edges, etc., since the dimension might change with the exact geometric structure of $\Delta$ (cf. [3], [4], [5]). Hence, sharp lower and upper bounds are important (cf. [6], [7]). The paper is devoted to the study of $\operatorname{dim} \hat{S}_{d}^{r}$, the dimension of the superspline subspace $\hat{S}_{d}^{r}$ of $S_{d}^{r}$. In particular, we will derive lower and upper bounds for $\operatorname{dim} \hat{S}_{d}^{r}(\Delta)$ for an arbitrary rectilinear grid partition $\Delta$. If $\Delta$ is a quasi-crosscut partition as introduced in [3], we will show that the dimension of $\hat{S}_{d}^{r}$ is given by the lower bound value. Here, a quasi-crosscut partition is a rectilinear partition which consists only of rays and crosscuts. Here, a ray is a straight line that starts at an interior vertex of $\Delta$ and terminates at a boundary vertex, and a crosscut is a straight line that joins two boundary vertices, and hence, divides the region $\Omega$ into two components. We will also show that for a type-1 triangulation $\Delta$ (that is, a triangulation obtained by drawing in all diagonals of positive slopes on a not necessarily uniform rectangular partition), the lower bound value also gives the exact dimension provided that $d>3 r$, and that for a type-2 triangulation $\Delta$ (that is, a triangulation obtained by drawing in both diagonals of each rectangular component of an arbitrary rectangular partition), the same conclusion holds provided that $d>[(8 r+1) / 3]$.

The proofs in this paper can be easily extended to study the dimension of the more general superspline space $S_{d}^{r, \rho}$ of functions in $S_{d}^{r}$ which have all partial derivatives of order $\rho$ at each (interior or boundary) vertex of $\Delta$. These results will be stated in Section 6. The notion of $S_{d}^{r, \rho}$ was introduced by Schumaker [8], where the dimension of this space is determined for the special case $d \geq 1+\max (2 \rho, 4 r)$. Note that $S_{d}^{r, r}=S_{d}^{r}$ and $S_{d}^{r, 2 r}=\hat{S}_{d}^{r}$.

2. Main Results. Let $E_{I}$ denote the number of interior edges and $V_{I}$ the number of interior vertices of an arbitrary rectilinear grid partition $\Delta$ of a closed simply connected polygon $\Omega$. Label the vertices $A_{1}, \ldots, A_{V_{I}}$ in some order, and let $e_{i}$ be the number of edges with different slopes attached to $A_{i}$. In addition, for each $i=1, \ldots, V_{I}$, denote by $\tilde{e}_{i}$ the number of edges with different slopes attached to $A_{i}$ but not $A_{j}, j<i$. Of course $\tilde{e}_{i}$ depends on the ordering of the vertices. The notation here follows that introduced by Schumaker [7] in the study of upper bounds of $\operatorname{dim} S_{d}^{r}$. Also, set

$$
N_{i}=\sum_{j=r+1}^{d-r}\left(r+j+1-(d-2 r) e_{i}\right)_{+}
$$

and

$$
\tilde{N}_{i}=\sum_{j=r+1}^{d-r}\left(r+j+1-(d-2 r) \tilde{e}_{i}\right)_{+} .
$$

We have the following result.

THEOREM 1. For $0 \leq d \leq 2 r$,

$$
\operatorname{dim} \hat{S}_{d}^{r}(\Delta)=\left(\begin{array}{c}
d+2 \\
2
\end{array}\right)
$$


the dimension of $\pi_{d}$. Let

$$
\begin{aligned}
D_{d}^{r}= & \operatorname{dim} \hat{S}_{d}^{r}(\Delta)-\left(\begin{array}{c}
d+2 \\
2
\end{array}\right) \\
= & E_{I}\left[\left(\begin{array}{c}
d-r+1 \\
2
\end{array}\right)-2\left(\begin{array}{c}
r+1 \\
2
\end{array}\right)+\left(\begin{array}{c}
\left.(3 r-d+1)_{+}\right) \\
2
\end{array}\right)\right] \\
& +V_{I}\left[\left(\begin{array}{c}
d+2 \\
2
\end{array}\right)-\left(\begin{array}{c}
2 r+2 \\
2
\end{array}\right)\right] .
\end{aligned}
$$

Then for $d>2 r \geq 0$,

$$
\sum_{i=1}^{V_{I}} N_{i} \leq D_{d}^{r} \leq \sum_{i=1}^{V_{I}} \tilde{N}_{i}
$$

Suppose now that the rectilinear grid partition is a quasi-crosscut partition $\Delta_{c}$ consisting of $L$ crosscuts. Let $l_{i}$ denote the number of crosscuts or rays that pass through or initiate from $A_{i}, i=1, \ldots, V_{I}$. We have the following result.

THEOREM 2. Let

$$
E_{d}^{r}:=\left[\left(\begin{array}{c}
d-r+1 \\
2
\end{array}\right)-2\left(\begin{array}{c}
r+1 \\
2
\end{array}\right)+\left(\begin{array}{c}
\left(r-(d-2 r)_{+}+1\right)_{+} \\
2
\end{array}\right)\right]_{+}
$$

and

$$
\begin{aligned}
F_{d}^{r}(n):=n & {\left[\left(\begin{array}{c}
d-r+1 \\
2
\end{array}\right)-2\left(\begin{array}{c}
r+1 \\
2
\end{array}\right)+\left(\begin{array}{c}
\left.r-(d-2 r)_{+}+1\right)_{+} \\
2
\end{array}\right)\right]_{+} } \\
& +\sum_{j=r+1}^{d-r}(r+j+1-n(d-2 r))_{+}-\left\{\left(\begin{array}{c}
d+2 \\
2
\end{array}\right)-\left(\begin{array}{c}
2 r+2 \\
2
\end{array}\right)\right\}_{+} .
\end{aligned}
$$

Then

$$
\operatorname{dim} \hat{S}_{d}^{r}\left(\Delta_{c}\right)=\left(\begin{array}{c}
d+2 \\
2
\end{array}\right)+L E_{d}^{r}+\sum_{i=1}^{V_{I}} F_{d}^{r}\left(l_{i}\right) .
$$

In particular, for $d \leq 2 r$,

$$
\operatorname{dim} \hat{S}_{d}^{r}\left(\Delta_{c}\right)=\left(\begin{array}{c}
d+2 \\
2
\end{array}\right)
$$

the dimension of $\pi_{d}$.

Next, we consider the following important consequences of these two results. Let

$$
\Omega_{R}=[a, b] \times[c, d]
$$

and $a=x_{0}<\cdots<x_{m+1}=b, c=y_{0}<\cdots<y_{n+1}=d$. Partition $\Omega_{R}$ first by drawing in the vertical lines $x-x_{i}=0$ and horizontal lines $y-y_{j}=0, i=1, \ldots, m$ and $j=1, \ldots, n$. Then by drawing in the diagonals with positive slopes to the rectangles $\left[x_{i}, x_{i+1}\right] \times\left[y_{j}, y_{j+1}\right]$, we obtain a type-1 triangulation $\Delta_{m n}^{(1)}$ of $\Omega_{R}$; and by drawing in both diagonals to each $\left[x_{i}, x_{i+1}\right] \times\left[y_{j}, y_{j+1}\right]$, we obtain a type-2 triangulation $\Delta_{m n}^{(2)}$ of $\Omega_{R}$. The triangulations $\Delta_{m n}^{(1)}$ and $\Delta_{m n}^{(2)}$ will be said to be uniform if $x_{i+1}-x_{i}=x_{i}-x_{i-1}$ and $y_{j+1}-y_{j}=y_{j}-y_{j-1}$ for all $i=1, \ldots, m$ and $j=1, \ldots, n$. As a consequence of Theorem 1, we have the following two results. 
COROLlaRY 1. Let $d>3 r$. Then

$$
\begin{aligned}
\operatorname{dim} \hat{S}_{d}^{r}\left(\Delta_{m n}^{(1)}\right)= & m n\left[d^{2}-3 r d+r(r-3) / 2\right] \\
& +(m+n)\left[d^{2}-(2 r-1) d-r^{2}-3 r\right] \\
& +d^{2}-(r-2) d-\left(r^{2}+3 r-2\right) / 2
\end{aligned}
$$

COROLlaRY 2. Let $d>[(8 r+1) / 3]$. Then

$$
\begin{aligned}
& \operatorname{dim} \hat{S}_{d}^{r}\left(\Delta_{m n}^{(2)}\right) \\
& =m n\left[6\left(\begin{array}{c}
d-r+1 \\
2
\end{array}\right)-12\left(\begin{array}{c}
r+1 \\
2
\end{array}\right)+6\left(\begin{array}{c}
(3 r-d+1)_{+} \\
2
\end{array}\right)\right. \\
& \left.-2\left(\begin{array}{c}
d+2 \\
2
\end{array}\right)+2\left(\begin{array}{c}
2 r+2 \\
2
\end{array}\right)+\sum_{j=r+1}^{d-r}(r+j+1-2(d-2 r))_{+}\right] \\
& +(m+n)\left[5\left(\begin{array}{c}
d-r+1 \\
2
\end{array}\right)-10\left(\begin{array}{c}
r+1 \\
2
\end{array}\right)+5\left(\begin{array}{c}
(3 r-d+1)_{+} \\
2
\end{array}\right)\right. \\
& \left.-\left(\begin{array}{c}
d+2 \\
2
\end{array}\right)+\left(\begin{array}{c}
2 r+2 \\
2
\end{array}\right)+\sum_{j=r+1}^{d-r}(r+j+1-2(d-2 r))_{+}\right] \\
& +\left(\begin{array}{c}
2 r+2 \\
2
\end{array}\right)+4\left(\begin{array}{c}
d-r+1 \\
2
\end{array}\right)-8\left(\begin{array}{c}
r+1 \\
2
\end{array}\right)+4\left(\begin{array}{c}
(3 r-d+1)_{+} \\
2
\end{array}\right) \\
& +\sum_{j=r+1}^{d-r}[r+j+1-2(d-2 r)]_{+} \text {. }
\end{aligned}
$$

When $\Delta_{m n}^{(1)}$ and $\Delta_{m n}^{(2)}$ are uniform type-1 and type-2 triangulations, respectively, they become quasi-crosscut partitions of $\Omega_{R}$ consisting only of crosscuts. Hence, Theorem 2 applies, and there are no longer any restrictions on the values of $d$ and $r$ as follows.

COROLlaRY 3. Let $\Delta_{m n}^{(1)}$ be a uniform type-1 triangulation of $\Omega_{R}$. Then for $d \geq 2 r$,

$$
\begin{aligned}
\operatorname{dim} \hat{S}_{d}^{r}\left(\Delta_{m n}^{(1)}\right) & \\
=\left(\begin{array}{c}
d+2 \\
2
\end{array}\right)+m n & {\left[3\left(\left(\begin{array}{c}
d-r+1 \\
2
\end{array}\right)-2\left(\begin{array}{c}
r+1 \\
2
\end{array}\right)+\left(\begin{array}{c}
\left.3 r-d+1)_{+}\right) \\
2
\end{array}\right)\right)\right.} \\
& \left.-\left(\begin{array}{c}
d+2 \\
2
\end{array}\right)+\left(\begin{array}{c}
2 r+2 \\
2
\end{array}\right)+\sum_{j=r+1}^{d-r}(r+j+1-3(d-2 r))_{+}\right] \\
+(2 m+2 n+1) & {\left[\left(\begin{array}{c}
d-r+1 \\
2
\end{array}\right)-2\left(\begin{array}{c}
r+1 \\
2
\end{array}\right)+\left(\begin{array}{c}
\left.(3 r-d+1)_{+}\right) \\
2
\end{array}\right)\right] . }
\end{aligned}
$$


In addition, for $d \leq 2 r$,

$$
\operatorname{dim} \hat{S}_{d}^{r}\left(\Delta_{m n}^{(1)}\right)=\left(\begin{array}{c}
d+2 \\
2
\end{array}\right)
$$

COROLLARY 4. Let $\Delta_{m n}^{(2)}$ be a uniform type-2 triangulation of $\Omega_{R}$. Then for $d \geq 2 r$

$$
\begin{aligned}
& \operatorname{dim} \hat{S}_{d}^{r}\left(\Delta_{m n}^{(2)}\right) \\
& =\left(\begin{array}{c}
d+2 \\
2
\end{array}\right)+m n\left[6\left(\begin{array}{c}
d-r+1 \\
2
\end{array}\right)-12\left(\begin{array}{c}
r+1 \\
2
\end{array}\right)\right. \\
& +6\left(\begin{array}{c}
(3 r-d+1)_{+} \\
2
\end{array}\right)-2\left(\begin{array}{c}
d+2 \\
2
\end{array}\right)+2\left(\begin{array}{c}
2 r+2 \\
2
\end{array}\right) \\
& +\sum_{j=r+1}^{d-r}(r+j-1-2(d-2 r))_{+} \\
& \left.+\sum_{j=r+1}^{d-r}(r+j-1-4(d-2 r))_{+}\right] \\
& +(m+n)\left[5\left(\begin{array}{c}
d-r+1 \\
2
\end{array}\right)-10\left(\begin{array}{c}
r+1 \\
2
\end{array}\right)+5\left(\begin{array}{c}
(3 r-d+1)_{+} \\
2
\end{array}\right)\right. \\
& \left.-\left(\begin{array}{c}
d+2 \\
2
\end{array}\right)+\left(\begin{array}{c}
2 r+2 \\
2
\end{array}\right)+\sum_{j=r+1}^{d-r}(r+j+1-2(d-2 r))_{+}\right] \\
& +\left[\left(\begin{array}{c}
2 r+2 \\
2
\end{array}\right)+4\left(\begin{array}{c}
d-r+1 \\
2
\end{array}\right)-8\left(\begin{array}{c}
r+1 \\
2
\end{array}\right)-\left(\begin{array}{c}
d+2 \\
2
\end{array}\right)\right. \\
& \left.+4\left(\begin{array}{c}
(3 r-d+1)_{+} \\
2
\end{array}\right)+\sum_{j=r+1}^{d-r}(r+j+1-2(d-2 r))_{+}\right] .
\end{aligned}
$$

In addition, for $d \leq 2 r$,

$$
\operatorname{dim} \hat{S}_{d}^{r}\left(\Delta_{m n}^{(2)}\right)=\left(\begin{array}{c}
d+2 \\
2
\end{array}\right)
$$

3. Key Lemma. We will first derive the dimension formula for the superspline space $\hat{S}_{d}^{r}\left(\Delta_{0}\right)$, where $\Delta_{0}$ is a rectilinear grid partition of $\Omega$ consisting of exactly one interior vertex $A$ such that all the interior edges of $\Delta_{0}$ connect $A$ to the boundary of $\Omega$. Let $\overline{A_{i} A}, i=1, \ldots, n_{0}$, be these edges arranged in the counterclockwise direction, where $A_{1}, \ldots, A_{n}$ are boundary vertices of $\Delta_{0}$, and let $N_{0}$ be the number of edges among $\overline{A_{1} A}, \ldots, \overline{A_{n_{0}} A}$ having different slopes. In Figure 1, for example, $n_{0}=6$ and $N_{0}=4$, and there are 11 boundary vertices, where those not connected to $A$ are not labelled. Denote by $\Omega_{i}, i=1, \ldots, n_{0}$, the polygonal component whose boundary consists of both of the edges $\overline{A_{i} A}$ and $\overline{A_{i-1} A}$, where $A_{0}:=A_{n_{0}}$. The following lemma will be used in the proofs of Theorems 1 and 2 . 


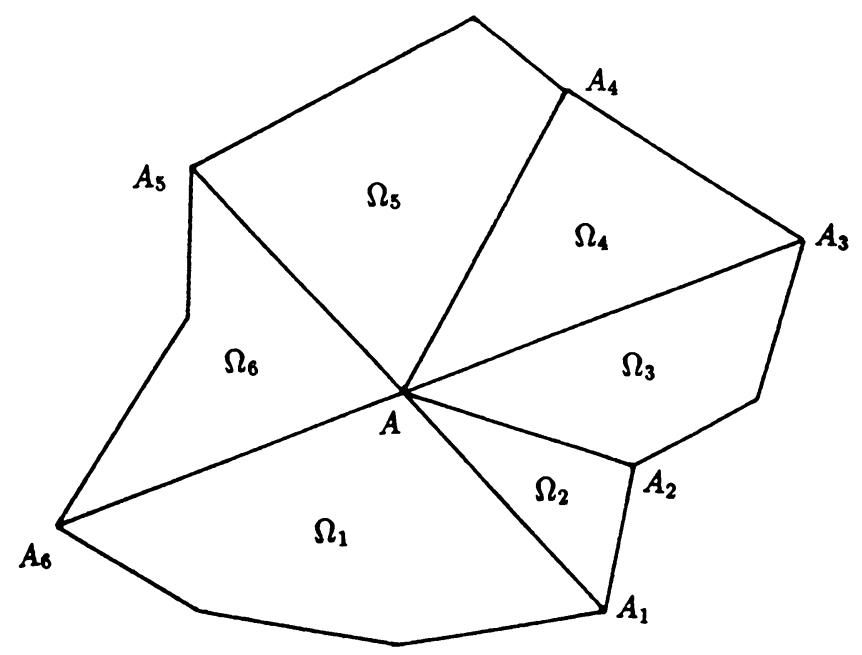

FIGURE 1

LEMMA 1. Let $\Delta_{0}$ be a rectilinear grid partition as described above. Then (13)

$$
\begin{aligned}
\operatorname{dim} \hat{S}_{d}^{r}\left(\Delta_{0}\right) & =\left(\begin{array}{c}
d+2 \\
2
\end{array}\right)+n_{0}\left[\left(\begin{array}{c}
d-r+1 \\
2
\end{array}\right)-2\left(\begin{array}{c}
r+1 \\
2
\end{array}\right)+\left(\begin{array}{c}
\left.\left(r-(d-2 r)_{+}+1\right)_{+}\right) \\
2
\end{array}\right)\right]_{+} \\
& +\sum_{j=r+1}^{d-r}\left[r+j+1-N_{0}(d-2 r)\right]_{+}-\left\{\left(\begin{array}{c}
d+2 \\
2
\end{array}\right)-\left(\begin{array}{c}
2 r+2 \\
2
\end{array}\right)\right\}_{+} .
\end{aligned}
$$

In particular, if $0 \leq d \leq 2 r$, then

$$
\operatorname{dim} \hat{S}_{d}^{r}\left(\Delta_{0}\right)=\left(\begin{array}{c}
d+2 \\
2
\end{array}\right)
$$

if $2 r<d \leq 3 r$, then

$$
\begin{aligned}
\operatorname{dim} \hat{S}_{d}^{r}\left(\Delta_{0}\right)= & \left(\begin{array}{c}
2 r+2 \\
2
\end{array}\right)+n_{0}\left[\left(\begin{array}{c}
d-r+1 \\
2
\end{array}\right)-2\left(\begin{array}{c}
r+1 \\
2
\end{array}\right)+\left(\begin{array}{c}
3 r-d+1 \\
2
\end{array}\right)\right] \\
& +\sum_{j=r+1}^{d-r}\left[r+j+1-N_{0}(d-2 r)\right]_{+}
\end{aligned}
$$

if $3 r<d \leq 4 r$, then

$$
\begin{aligned}
\operatorname{dim} \hat{S}_{d}^{r}\left(\Delta_{0}\right)= & \left(\begin{array}{c}
2 r+2 \\
2
\end{array}\right)+n_{0}\left[\left(\begin{array}{c}
d-r+1 \\
2
\end{array}\right)-2\left(\begin{array}{c}
r+1 \\
2
\end{array}\right)\right] \\
& +\sum_{j=r+1}^{d-r}\left[r+j+1-N_{0}(d-2 r)\right]_{+}
\end{aligned}
$$

and if $d>4 r$, then

$$
\operatorname{dim} \hat{S}_{d}^{r}\left(\Delta_{0}\right)=\left(\begin{array}{c}
2 r+2 \\
2
\end{array}\right)+n_{0}\left[\left(\begin{array}{c}
d-r+1 \\
2
\end{array}\right)-2\left(\begin{array}{c}
r+1 \\
2
\end{array}\right)\right] .
$$

Proof. Without loss of generality, we assume that the interior vertex $A$ is located at the origin and that the equation of each edge $\overline{A_{i} A}, i=1, \ldots, n_{0}$, is given by 
$y+\alpha_{i} x=0$, where $\alpha_{i} \neq 0$. Let $s \in \hat{S}_{d}^{r}\left(\Delta_{0}\right)$, and set $p_{i}:=\left.s\right|_{\Omega_{i}}-\left.s\right|_{\Omega_{1}}$. Hence, $p_{i} \in \pi_{d}$ with $p_{1} \equiv 0$. Following [6], we write

$$
p_{i}:=\sum_{j=1}^{d-r} \sum_{k=1}^{j} c_{i-1, j k} \phi_{j k}^{i-1},
$$

where

$$
\phi_{j k}^{i}(x, y):=x^{j-k}\left(y+\alpha_{i} x\right)^{r+k} .
$$

Since $p_{1} \equiv 0$ and $s \in \hat{S}_{d}^{r}\left(\Delta_{0}\right)$, we have, for each $i=2, \ldots, n_{0}$,

$$
\frac{\partial^{r+m}}{\partial y^{l} \partial x^{r+m-l}} p_{i}(0)=0
$$

where $l=0, \ldots, r+m$ and $m=1, \ldots, r$, so that the representation of $p_{i}$ in (15) becomes

$$
p_{i}=\sum_{j=r+1}^{d-r} \sum_{k=1}^{j} c_{i-1, j k} \phi_{j k}^{i-1}
$$

If $d \leq 2 r$, then it is clear that $p_{i} \equiv 0, i=2, \ldots, n_{0}$, so that

$$
\left.s \equiv s\right|_{\Omega_{1}} \in \pi_{d}
$$

and this proves the conclusion in (14). Hence, we may now assume that $d>2 r$, and under this assumption, (13) can be simplified to be

$$
\begin{aligned}
\operatorname{dim} & \hat{S}_{d}^{r}\left(\Delta_{0}\right) \\
= & \left(\begin{array}{c}
2 r+2 \\
2
\end{array}\right)+n_{0}\left[\left(\begin{array}{c}
d-r+1 \\
2
\end{array}\right)-2\left(\begin{array}{c}
r+1 \\
2
\end{array}\right)+\left(\begin{array}{c}
\left.3 r-d+1)_{+}\right) \\
2
\end{array}\right)\right] \\
& +\sum_{j=r+1}^{d-r}\left[r+j+1-N_{0}(d-2 r)\right]_{+} .
\end{aligned}
$$

We are now ready to apply the "conformality condition" (cf. [1]) of $s$ at 0 by using the representations of the polynomial pieces $p_{i}$ in (16), yielding the following homogeneous system of $(d-2 r)(d+2 r+3) / 2$ linear equations in $n_{0}\left[\left(\begin{array}{c}d-r+1 \\ 2\end{array}\right)-\left(\begin{array}{c}r+1 \\ 2\end{array}\right)\right]$ unknowns:

$$
H \mathbf{c}=\mathbf{0},
$$

where

$$
\begin{aligned}
\mathbf{c} & =\left(\mathbf{c}_{r+1}^{T}, \ldots, \mathbf{c}_{d-r}^{T}\right)^{T} \\
\mathbf{c}_{j} & :=\left(c_{1 j j}, \ldots, c_{1 j 1}, \ldots, c_{n_{0} j j}, \ldots, c_{n_{0} j 1}\right)^{T}
\end{aligned}
$$

and

$$
H=\left[\begin{array}{llll}
H_{r+1} & & & \\
& H_{r+2} & & \\
& & \ddots & \\
& & & H_{d-r}
\end{array}\right]
$$


Here, for each $j=r+1, \ldots, d-r, H_{j}$ is an $(r+j+1) \times\left(n_{0} j\right)$ matrix of the form $H_{j}=\left[H_{j 1} \cdots H_{j n_{0}}\right]$ with

$$
H_{j i}=\left[\begin{array}{cccc}
1 & & & \\
\left(\begin{array}{c}
r+j \\
1
\end{array}\right) \alpha_{i} & 1 & . & \\
\left(\begin{array}{c}
r+j \\
2
\end{array}\right) \alpha_{i}^{2} & \left(\begin{array}{c}
r+j-1 \\
1
\end{array}\right) \alpha_{i} & & 1 \\
\vdots & \vdots & & \vdots \\
\left(\begin{array}{c}
r+j \\
r+j
\end{array}\right) \alpha_{i}^{r+j} & \left(\begin{array}{c}
r+j-1 \\
r+j-1
\end{array}\right) \alpha_{i}^{r+j-1} & \ldots & \left(\begin{array}{c}
r+1 \\
r+1
\end{array}\right) \alpha_{i}^{r+1}
\end{array}\right], \quad i=1, \ldots, n_{0}
$$

Before studying (18), we still have to incorporate the $C^{2 r}$ condition of the spline function $s$ at $A_{1}, \ldots, A_{n_{0}}$. That is, the polynomials $p_{i}, i=1, \ldots, n_{0}$, with $P_{n_{0}+1}:=$ $p_{1} \equiv 0$, must satisfy

$$
\frac{\partial^{r+m}}{\partial y^{l} \partial x^{r+m-l}}\left(p_{i+1}-p_{i}\right)\left(A_{i}\right)=0
$$

for $l=0, \ldots, r+m, m=1, \ldots, r$, and $i=1, \ldots, n_{0}$. Using the formulation of $p_{i}$ in (16), it is clear that (19) becomes

$$
\begin{aligned}
& \sum_{j=r+1}^{d-r} x_{i}^{j} c_{i j k}=0, \\
& \sum_{j=r+1}^{d-r}(j-k) x_{i}^{j} c_{i j k}=0, \\
& \quad \cdots \cdots \cdots \\
& \sum_{j=r+1}^{d-r}(j-k) \cdots(j-r+1) x_{i}^{j} c_{i j k}=0,
\end{aligned}
$$

where $k=1, \ldots, r$ and $i=1, \ldots, n_{0}$; and in matrix form:

$$
\begin{gathered}
Q_{0} \mathbf{c}_{i k}=\mathbf{0} \\
\mathbf{c}_{i k}:=\left(c_{i, r+1, k}, c_{i, r+2, k}, \ldots, c_{i, d-r, k}\right)^{r}
\end{gathered}
$$

and

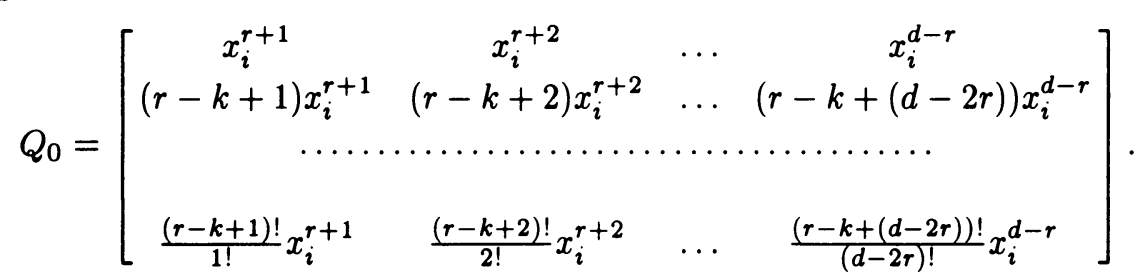

We now apply elementary row operations to the $(r-k+1) \times(d-2 r)$ matrix $Q_{0}$ as follows. For $l=r-k, \ldots, 3,2$ consecutively, we add the $(l-1)$ multiple of the $l$ th row to the $(l+1)$ st row of $Q_{0}$ yielding $Q_{1}$. Next, for $l=r-k, \ldots, 4,3$ consecutively, we add the $(l-2)$ multiple of the $l$ th row to the $(l+1)$ st row of $Q_{1}$ yielding $Q_{2}$, etc. This procedure is performed until $Q_{r-k-1}$ is obtained, and Eq. (20) is now reduced to

$$
Q_{r-k-1} \mathbf{c}_{i k}=\mathbf{0}
$$


where

$$
Q_{r-k-1}=\left[\begin{array}{cccc}
x_{i}^{r+1} & x_{i}^{r+2} & \ldots & x_{i}^{d-r} \\
(r-k+1) x_{i}^{r+1} & (r-k+2) x_{i}^{r+2} & \ldots & (d-r-k) x_{i}^{d-r} \\
(r-k+1)^{2} x_{i}^{r+1} & (r-k+2)^{2} x_{i}^{r+2} & \ldots & (d-r-k)^{2} x_{i}^{d-r} \\
\ldots \ldots \ldots \ldots \ldots \ldots \ldots \ldots \ldots \ldots \ldots \ldots \ldots \ldots \ldots \ldots \ldots \ldots \\
(r-k+1)^{r-k} x_{i}^{r+1} & (r-k+2)^{r-k} x_{i}^{r+2} & \ldots & (d-r-k)^{r-k} x_{i}^{d-r}
\end{array}\right] .
$$

It is clear that $Q_{r-k-1}$ has full rank; that is,

$$
q_{k}:=\operatorname{rank} Q_{r-k-1}=r-k+1-(3 r-k-d+1)_{+} .
$$

Hence, Eq. (20) or (21) is equivalent to the linear relation

$$
c_{i j k}=\sum_{l=r+1}^{d-r-q_{k}} \delta_{i l k}^{j} c_{i l k}
$$

where $j=d-r-q_{k}+1, \ldots, d-r ; k=1, \ldots, r$; and $i=1, \ldots, n_{0}$. Note that if $d-r-q_{k} \leq r$, then the summation in (22) is an empty sum, and these $c_{i j k}$ are all equal to zero. In any case, the relation in (22) can be substituted into the linear system (18) to yield the following system of linear equations:

$$
H^{\prime} \mathbf{c}^{\prime}=\mathbf{0},
$$

where $\mathbf{c}^{\prime}=\left(\mathbf{c}_{r+1}^{\prime T}, \ldots, \mathbf{c}_{d-r}^{\prime T}\right)^{T}$ with $\mathbf{c}_{j}^{\prime}=\mathbf{c}_{j}$ for $j=r+1, \ldots, d-2 r$ as in (18), and for $j=d-2 r+1, \ldots, d-r$,

$$
\mathbf{c}_{j}^{\prime}=\left(c_{1, j, j}, \ldots, c_{1, j, 2 r+j-d+1}, \ldots, c_{n_{0}, j, j}, \ldots, c_{n_{0}, j, 2 r+j-d+1}\right) .
$$

The formulation of $H^{\prime}$ depends on whether $d \geq 3 r+1$ or $2 r<d \leq 3 r$. (Recall that for $0 \leq d \leq 2 r$, we have already established (14).)

Case (i). Let $d \geq 3 r+1$. Then

$$
H^{\prime}=\left[\begin{array}{cccccc}
H_{r+1} & & 0 & & & \\
& & & & & \\
0 & \ddots & & & & \\
B_{r+1}^{d-2 r+1} & \ldots & B_{d-2 r}^{d-2 r+1} & \bar{H}_{d-2 r+1} & & \\
\vdots & & \vdots & \ddots & \ddots & \\
B_{r+1}^{d-r} & \ldots & B_{d-2 r}^{d-r} & \ldots & B_{d-r-1}^{d-r} & \bar{H}_{d-r}
\end{array}\right],
$$

where $H_{r+1}, \ldots, H_{d-2 r}$ have been introduced in defining the coefficient matrix $H$ in (18). The other block submatrices in $H^{\prime}$ are defined as follows:

$$
\bar{H}_{j}=\left[\bar{H}_{j 1} \cdots \bar{H}_{j n_{0}}\right] \text {, }
$$

where $\bar{H}_{j i}=\left[h_{u v}\right]$ is $(r+j+1) \times(j-\mu)$, with $\mu:=2 r+j-d$, and

$$
h_{u v}= \begin{cases}\left(\begin{array}{c}
r+j-v \\
u-v
\end{array}\right) \alpha_{i}^{u-v} & \text { for } 0 \leq v \leq u \leq r+j, \\
0 & \text { for } 0 \leq u<v \leq j-\mu-1 ;\end{cases}
$$

and $B_{l}^{j}=\left[B_{l 1}^{j} \cdots B_{l n_{0}}^{j}\right]$, where $B_{l i}^{j}$ is an $(r+j+1) \times l$ block defined by

$$
B_{l i}^{j}=\left[\begin{array}{cc}
0 & 0 \\
0 & \hat{B}_{l i}^{j}
\end{array}\right]
$$


with $\hat{B}_{l i}^{j}=\left[b_{u v}^{j}\right]$ being an $(r+\mu+1) \times \mu$ block with entries

$$
b_{i v}^{j}= \begin{cases}\delta_{i, l, \mu-v}^{j}\left(\begin{array}{c}
r+\mu-v \\
u-v
\end{array}\right) \alpha_{i}^{u-v} & \text { for } 0 \leq v \leq u \leq r+\mu, \\
0 & \text { for } 0 \leq u<v \leq \mu-1 .\end{cases}
$$

Case (ii). Let $2 r<d \leq 3 r$. Then

$$
H^{\prime}=\left[\begin{array}{ccccc}
\bar{H}_{r+1} & & & & \\
D_{r+1}^{r+2} & \bar{H}_{r+2} & & 0 & \\
\vdots & \ddots & \cdot & . & \\
D_{r+1}^{d-r} & \ldots & D_{d-r-1}^{d-r} & & \bar{H}_{d-r}
\end{array}\right]
$$

where $\bar{H}_{r+1}, \ldots, \bar{H}_{d-r}$ have been introduced in case (i), and $D_{l}^{j}=\left[D_{l 1}^{j} \cdots D_{l n_{0}}^{j}\right]$ with $D_{l i}^{j}$ being an $(r+j+1) \times(j-\mu)$ block defined by

$$
D_{l i}^{j}=\left[\begin{array}{cc}
0 & 0 \\
0 & \hat{D}_{l i}^{j}
\end{array}\right]
$$

and $\hat{D}_{l i}^{j}=\left[d_{u v}^{j}\right]$ an $(r+\mu+1) \times(j-l)$ block with entries

$$
d_{u v}^{j}= \begin{cases}\delta_{i, l, r+\mu-l-v+1}^{j}\left(\begin{array}{c}
2 r+\mu-l-v+1 \\
u-v
\end{array}\right) \alpha_{i}^{u-v} & \text { for } 0 \leq v \leq u \leq r+\mu \\
0 & \text { for } 0 \leq u<v \leq j-l-1\end{cases}
$$

Note that in the entries of $\hat{B}_{l i}^{j}$ and $\hat{D}_{l i}^{j}$, the coefficients $\delta_{i, l, k}^{j}$ come from the linear relation (22).

To determine the dimension of the null space of the linear system (23), we will compute the rank of $H^{\prime}$. First, by referring to the proof of Theorem 2.1 in Schumaker [6], we have, for $j \geq r+1$,

$$
\operatorname{rank} H_{j}=\min \left(r+j+1, N_{0} j\right)=r+j+1
$$

and

$$
\begin{aligned}
\operatorname{rank} \bar{H}_{j} & =\min \left(r+j+1, N_{0}(d-2 r)\right) \\
& =r+j+1-\left[r+j+1-N_{0}(d-2 r)\right]_{+} .
\end{aligned}
$$

Hence, for $d \geq 3 r+1$, we go to case (i) and conclude that

$$
\begin{aligned}
\operatorname{rank} H^{\prime} & =\sum_{j=r+1}^{d-2 r} \operatorname{rank} H_{j}+\sum_{j=d-2 r+1}^{d-r} \operatorname{rank} \bar{H}_{j} \\
& =\sum_{j=r+1}^{d-2 r}(r+j+1)+\sum_{j=d-2 r+1}^{d-r}\left(r+j+1-\left[r+j+1-N_{0}(d-2 r)\right]_{+}\right) \\
& =\sum_{j=r+1}^{d-r}(r+j+1)-\sum_{j=d-2 r+1}^{d-r}\left[r+j+1-N_{0}(d-2 r)\right]_{+} \\
& =\left(\begin{array}{c}
d+2 \\
2
\end{array}\right)-\left(\begin{array}{c}
2 r+2 \\
2
\end{array}\right)-\sum_{j=r+1}^{d-r}\left[r+j+1-N_{0}(d-2 r)\right]_{+},
\end{aligned}
$$

where the lower limit of summation is changed from $d-2 r+1$ to $r+1$ since $N_{0} \geq 2$ and $d \geq 3 r+1$. 
For $2 r<d \leq 3 r$, we go to case (ii) and conclude that

$$
\begin{aligned}
\operatorname{rank} H^{\prime} & =\sum_{j=r+1}^{d-r} \operatorname{rank} \bar{H}_{j} \\
& =\sum_{j=r+1}^{d-r}\left((r+j+1)-\left[r+j+1-N_{0}(d-2 r)\right]_{+}\right) \\
& =\left(\begin{array}{c}
d+2 \\
2
\end{array}\right)-\left(\begin{array}{c}
2 r+2 \\
2
\end{array}\right)-\sum_{j=r+1}^{d-r}\left[r+j+1-N_{0}(d-2 r)\right]_{+},
\end{aligned}
$$

which is identical to (24). That is, for all $d>2 r$, by unifying cases (i) and (ii), the null space of the matrix transformation $H^{\prime}$ (or equivalently the solution space of (23)) has dimension

$$
\begin{aligned}
n_{0}\left[\left(\begin{array}{c}
d-r+1 \\
2
\end{array}\right)-2\left(\begin{array}{c}
r+1 \\
2
\end{array}\right)+\left(\begin{array}{c}
(3 r-d+1)_{+} \\
2
\end{array}\right)\right]-\operatorname{rank} H^{\prime} \\
=\left(\begin{array}{c}
2 r+2 \\
2
\end{array}\right)+n_{0}\left[\left(\begin{array}{c}
d-r+1 \\
2
\end{array}\right)-2\left(\begin{array}{c}
r+1 \\
2
\end{array}\right)+\left(\begin{array}{c}
\left.(3 r-d+1)_{+}\right) \\
2
\end{array}\right)\right] \\
\quad+\sum_{j=r+1}^{d-r}\left[r+j+1-N_{0}(d-2 r)\right]_{+}-\left(\begin{array}{c}
d+2 \\
2
\end{array}\right) .
\end{aligned}
$$

This is the number of free parameters in $s-\left.s\right|_{\Omega_{1}}$, where $\left.s\right|_{\Omega_{1}} \in \pi_{d}$, for any superspline function $s \in \hat{S}_{d}^{r}\left(\Delta_{0}\right), d>2 r$. Hence, we have established the formula (17), and therefore (13) for any $d$ and $r$, completing the proof of the lemma.

4. Proof of Theorem 1 and Its Consequences. Our proof of Theorem 1 will depend on the dimension formula for a rectilinear grid partition $\Delta_{1}$ which is somewhat more general than the grid partition $\Delta_{0}$ studied in the previous section. More precisely, let $\Delta_{1}$ consist of at most one interior vertex and $E_{I}$ interior edges, such that if $\Delta_{1}$ does contain an interior vertex $A$, then there are $n_{0}$ polygonal components $\Omega_{1}, \ldots, \Omega_{n_{0}}$ with $A$ as their common vertex, and the notation such as $A_{i}$ and $N_{0}$ introduced in Section 3 will also be used here. Note, in particular, that $E_{I} \geq n_{0}$, and two examples of $\Delta_{1}$ are illustrated in Figure 2: the one on the left without an interior vertex and the one on the right consisting of an interior vertex $A$. Let $\varepsilon=0$ or 1 denote the number of interior vertices of $\Delta_{1}$. We have the following lemma.
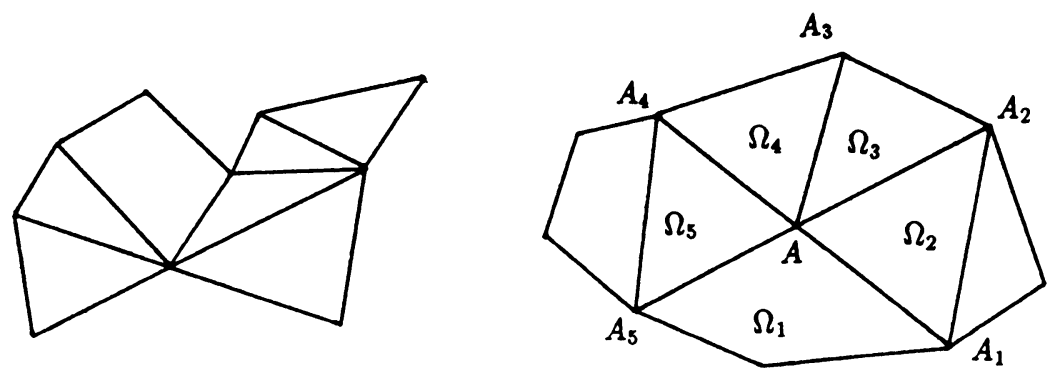

FIGURE 2 
LEMMA 2. Let $\Delta_{1}$ be a rectilinear grid partition as described above. Then

$$
\begin{aligned}
\operatorname{dim} & \hat{S}_{d}^{r}\left(\Delta_{1}\right) \\
= & \left(\begin{array}{c}
d+2 \\
2
\end{array}\right)+E_{I}\left[\left(\begin{array}{c}
d-r+1 \\
2
\end{array}\right)-2\left(\begin{array}{c}
r+1 \\
2
\end{array}\right)+\left(\begin{array}{c}
\left.\left(r-(d-2 r)_{+}+1\right)_{+}\right) \\
2
\end{array}\right)\right]_{+} \\
& -\varepsilon\left\{\left(\begin{array}{c}
d+2 \\
2
\end{array}\right)-\sum_{j=r+1}^{d-r}\left[r+j-1-N_{0}(d-2 r)\right]_{+}-\left(\begin{array}{c}
2 r+2 \\
2
\end{array}\right)\right\}_{+} .
\end{aligned}
$$

Proof. Let us first consider the case when $\varepsilon=1$, where there are $n_{1}:=E_{I}-n_{0}$ interior edges that are not attached to the interior vertex $A$. In other words, there are $n_{1}$ polygonal components which do not contain $A$ as a vertex so that the polynomial representations of any $s \in \hat{S}_{d}^{r}\left(\Delta_{1}\right)$ on these components are not governed by the conformality condition at $A$. However, they are specified by the "smoothing cofactors" across the $n_{1}$ edges (cf. [1]). Since the number of free parameters in each smoothing cofactor is $\left(\begin{array}{c}d-r+1 \\ 2\end{array}\right)$ and the "super" smoothness requirement at both vertices of the corresponding edge induces

$$
2\left(\begin{array}{c}
r+1 \\
2
\end{array}\right)-\left(\begin{array}{c}
\left(r-(d-2 r)_{+}+1\right)_{+} \\
2
\end{array}\right)
$$

linearly independent constraints (cf. the arguments in the derivation of (16) and the consideration of both cases (i) and (ii) in the proof of Lemma 1), we may conclude that the contribution to the dimension of $\hat{S}_{d}^{r}\left(\Delta_{1}\right)$ from the components not attached to $A$ is

$$
n_{1}\left[\left(\begin{array}{c}
d-r+1 \\
2
\end{array}\right)-2\left(\begin{array}{c}
r+1 \\
2
\end{array}\right)+\left(\begin{array}{c}
\left(r-(d-2 r)_{+}+1\right)_{+} \\
2
\end{array}\right)\right] .
$$

Adding this quantity to the quantity given in (13), which is of course the contribution to the dimension from the polygonal components attached to $A$, we have established (25) with $\varepsilon=1$. For $\varepsilon=0$, the proof is similar since the contribution to the dimension from one component is $\left(\begin{array}{c}d+2 \\ 2\end{array}\right)$ and the contribution from the other components is given by the number of free parameters allowed by the smoothing cofactors with constraints induced by the "super" smoothness requirement at the vertices. This completes the proof of Lemma 2 .

We now turn to the proof of Theorem 1. Following Schumaker [6], we will establish the lower bound formula in (5) by using mathematical induction. Recall that $\Delta$ is a rectilinear grid partition of a polygonal region $\Omega$. In view of Lemma 2 , we may assume that the number of interior vertices $V_{I}$ of $\Delta$ is at least 2 . Let $A_{0}$ be an interior vertex of $\Delta$ such that there exists at least one edge that connects $A_{0}$ to a boundary vertex. Let $\Omega_{1}^{1}$ be a closed polygonal subregion of $\Omega$ with grid partition $\Delta_{1}^{1}$ induced by $\Delta$ such that $A_{0}$ is an interior vertex, and the only one, of $\Delta_{1}^{1}$ and that the complement of the $\Omega_{1}^{1}$ with respect to $\Omega$ is also a polygonal region. In addition, let $\tilde{\Omega}$ be another closed polygonal subregion of $\Omega$ with grid partition $\tilde{\Delta}$ induced by $\Delta$ such that $\tilde{\Delta}$ has exactly $V_{I}-1$ interior vertices with $A_{0}$ as a boundary vertex and that the complement of $\tilde{\Omega}$ with respect to $\Omega$ is also a polygonal region. Let $\Omega_{1}^{0}=\tilde{\Omega} \cap \Omega_{1}^{1}$ with grid partition $\Delta_{1}^{0}$ induced by $\Delta$. Note that $\left(\Omega_{1}^{0}, \Delta_{1}^{0}\right)$ has no interior vertex while $\left(\Omega_{1}^{1}, \Delta_{1}^{1}\right)$ has exactly one interior vertex as illustrated by the 
two examples in Figure 2. Hence, the dimensions of $\hat{S}_{d}^{r}\left(\Delta_{1}^{0}, \Omega_{1}^{0}\right)$ and $\hat{S}_{d}^{r}\left(\Delta_{1}^{1}, \Omega_{1}^{1}\right)$ are given by formula (25) with $\varepsilon=0$ and 1, respectively. Note that the three spline spaces $\hat{S}_{d}^{r}(\tilde{\Delta}, \tilde{\Omega}), \hat{S}_{d}^{r}\left(\Delta_{1}^{1}, \Omega_{1}^{1}\right)$, and $\hat{S}_{d}^{r}\left(\Delta_{1}^{0}, \Omega_{1}^{0}\right)$ may be considered as subspaces of the original superspline space $\hat{S}_{d}^{r}(\Delta, \Omega)$ since each function in any of these spline spaces is the restriction of some function in $\hat{S}_{d}^{r}(\Delta, \Omega)$. Consequently,

$$
\hat{S}_{d}^{r}\left(\Delta_{1}^{0}, \Omega_{1}^{0}\right)=\hat{S}_{d}^{r}(\tilde{\Delta}, \tilde{\Omega}) \cap \hat{S}_{d}^{r}\left(\Delta_{1}^{1}, \Omega_{1}^{1}\right)
$$

so that

$$
\begin{aligned}
\operatorname{dim} \hat{S}_{d}^{r}(\Delta, \Omega) \geq & \operatorname{dim} \hat{S}_{d}^{r}(\tilde{\Delta}, \tilde{\Omega})+\operatorname{dim} \hat{S}_{d}^{r}\left(\Delta_{1}^{1}, \Omega_{1}^{1}\right) \\
& -\operatorname{dim} \hat{S}_{d}^{r}\left(\Delta_{1}^{0}, \Omega_{1}^{0}\right) .
\end{aligned}
$$

Let $\tilde{E}_{I}, E_{I}^{1}$, and $E_{I}^{0}$ be the number of interior edges of $\tilde{\Delta}, \Delta_{1}^{1}$, and $\Delta_{1}^{0}$, respectively. It can be verified that

$$
E_{I}=\tilde{E}_{I}+E_{I}^{1}-E_{I}^{0} .
$$

The proof of the lower bound result in (5) is therefore completed by applying the induction hypothesis to $\operatorname{din} 1 \hat{S}_{d}^{r}(\tilde{\Delta}, \tilde{\Omega})$ in $(26)$, using the dimension formula in Lemma 2 with both $\varepsilon=1$ and 0 , and appealing to (27).

The proof of the upper bound result in (5) is exactly the same as for Theorem 2.1 in Schumaker [7].

To prove Corollary 1 , where $\Delta_{m n}^{(1)}$ is an arbitrary type-1 triangulation of the rectangle $\Omega_{R}$, we order the interior vertices of $\Delta_{m n}^{(1)}$ as follows:

$$
\left(x_{1}, y_{1}\right), \ldots,\left(x_{1}, y_{n}\right),\left(x_{2}, y_{1}\right), \ldots,\left(x_{2}, y_{n}\right), \ldots,\left(x_{m}, y_{1}\right), \ldots,\left(x_{m}, y_{n}\right)
$$

so that $\tilde{e}_{i} \geq 3$ for all $i=1, \ldots, m n$. Hence, for $d \geq 3 r+1$, we have $((r+j+1)$ $\left.-e_{i}(d-2 r)\right) \leq 0$ for $j=r+1, \ldots, d-r$, and this implies that $\tilde{N}_{i}=0$ for all $i=1, \ldots, m n$; or $D_{d}^{r}=0$. This establishes (9).

For Corollary 2, where $\Delta_{m n}^{(2)}$ is an arbitrary type-2 triangulation of $\Omega_{R}$, we order the interior vertices as follows:

$$
\begin{array}{r}
\left(x_{1-1 / 2}, y_{1-1 / 2}\right), \ldots,\left(x_{1-1 / 2}, y_{n+1 / 2}\right), \ldots,\left(x_{1}, y_{1}\right), \ldots,\left(x_{1}, y_{n}\right), \\
\left(x_{1+1 / 2}, y_{1-1 / 2}\right), \ldots,\left(x_{1+1 / 2}, y_{n+1 / 2}\right), \ldots,\left(x_{m}, y_{1}\right), \ldots,\left(x_{m}, y_{n}\right), \\
\left(x_{m+1 / 2}, y_{1-1 / 2}\right), \ldots,\left(x_{m+1 / 2}, y_{n+1 / 2}\right),
\end{array}
$$

where $x_{i+1 / 2}:=\left(x_{i}+x_{i+1}\right) / 2$ and $y_{j+1 / 2}:=\left(y_{j}+y_{j+1}\right) / 2$. Hence, if $A_{i}=\left(x_{p_{i}}, y_{q_{i}}\right)$ where $p_{i}$ and $q_{i}$ are integers, then $\tilde{e}_{i} \geq 4$, so that for $d>[(8 r+1) / 3]$ we have $\tilde{N}_{i}=0$. On the other hand, if $A_{i}=\left(x_{p_{i}+1 / 2}, y_{q_{i}+1 / 2}\right)$, then $e_{i}=\tilde{e}_{i}=2$, so that $N_{i}=\tilde{N}_{i}$. That is, for $d>[(8 r+1) / 3]$, the upper and lower bounds for $D_{d}^{r}$ in (5) agree. Hence, the dimension formula in (10) follows from (4) by using (1).

5. Proof of Theorem 2 and Its Consequences. The procedure in our proof of Theorem 2 is identical to that of Theorem 1 in [3] (cf. [1] for more detail). We start from any interior vertex that connects to a boundary vertex and apply Corollary 1 to determine the contribution of the polygonal components around this interior vertex to $\operatorname{dim} \hat{S}_{d}^{r}\left(\Delta_{c}\right)$. Then we go along this ray or crosscut to the other interior vertices that lie on this line consecutively to account for their contributions to the dimension. If this line happens to be a crosscut, the smoothing cofactor across the 
final interior edge on this crosscut has an additional contribution to the dimension. The number of free parameters here is given by $E_{d}^{r}$ in (6) which is of course the same as the dimension of the vector $\mathbf{c}^{\prime}$ in (23). We next consider the second ray or crosscut and follow the same procedure. When all rays and crosscuts have been considered, we arrive at the dimension formula given by (8). This completes the proof of Theorem 2. To verify the dimension formulas in Corollaries 3 and 4, we simply apply Theorem 2 by counting the number of crosscuts and using $e_{i}=l_{i}=3$ in Corollary 3 and $e_{i}=l_{i}=2$ or 4 in Corollary 4 , respectively.

6. Extensions. After the first draft of this paper was written, we learnt that Schumaker [8] has perfected the notion of supersplines by introducing the subspace $S_{d}^{r, \rho}(\Delta)$ of $S_{d}^{r}(\Delta)$ of functions which have all partial derivatives of order at least $\rho$ at each vertex of $\Delta$. Note that $S_{d}^{r, r}(\Delta)=S_{d}^{r}(\Delta)$ and $S_{d}^{r, 2 r}(\Delta)=\hat{S}_{d}^{r}(\Delta)$. By a modification of the proofs in Sections 3, 4, and 5, the following results may be established. Let $r \leq \rho \leq d$ and set

(28) $N_{i}^{\rho}:=\sum_{j=\rho-r+1}^{d-\rho}\left(r+j+1-j e_{i}\right)_{+}+\sum_{j=1+\max (d-\rho, \rho-r)}^{d-r}\left(r+j+1-(d-\rho) e_{i}\right)_{+}$

and

(29) $\tilde{N}_{i}^{\rho}=\sum_{j=\rho-r+1}^{d-\rho}\left(r+j+1-j \tilde{e}_{i}\right)_{+}+\sum_{j=1+\max (d-\rho, \rho-r)}^{d-r}\left(r+j+1-(d-\rho) \tilde{e}_{i}\right)_{+} \cdot$

THEOREM 3. For $0 \leq d \leq \rho$,

$$
\operatorname{dim} S_{d}^{r, \rho}(\Delta)=\left(\begin{array}{c}
d+2 \\
2
\end{array}\right)
$$

Let

$$
\begin{aligned}
D_{d}^{r, \rho}= & \operatorname{dim} S_{d}^{r, \rho}(\Delta)-\left(\begin{array}{c}
d+2 \\
2
\end{array}\right) \\
& -E_{I}\left[\left(\begin{array}{c}
d-r+1 \\
2
\end{array}\right)-2\left(\begin{array}{c}
\rho-r+1 \\
2
\end{array}\right)+\left(\begin{array}{c}
\left.2 \rho-r-d+1)_{+}\right) \\
2
\end{array}\right)\right] \\
& +V_{I}\left[\left(\begin{array}{c}
d+2 \\
2
\end{array}\right)-\left(\begin{array}{c}
\rho+2 \\
2
\end{array}\right)\right]
\end{aligned}
$$

Then for $d>\rho$,

$$
\sum_{i=1}^{V_{I}} N_{i}^{\rho} \leq D_{d}^{r, \rho} \leq \sum_{i=1}^{V_{I}} \tilde{N}_{i}^{\rho}
$$

THEOREM 4. Let $r \leq \rho \leq d$,

$$
E_{d}^{r, \rho}:=\left[\left(\begin{array}{c}
d-r+1 \\
2
\end{array}\right)-2\left(\begin{array}{c}
\rho-r+1 \\
2
\end{array}\right)+\left(\begin{array}{c}
(2 \rho-r-d+1)_{+} \\
2
\end{array}\right)\right]_{+}
$$


and

$$
\begin{aligned}
F_{d}^{r, \rho}(n):= & n\left[\left(\begin{array}{c}
d-r+1 \\
2
\end{array}\right)-2\left(\begin{array}{c}
\rho-r+1 \\
2
\end{array}\right)+\left(\begin{array}{c}
\left.(2 \rho-r-d+1)_{+}\right) \\
2
\end{array}\right)\right]_{+} \\
& +\sum_{j=\rho-r+1}^{d-\rho}(r+j+1-j n)_{+}+\sum_{j=1+\max (d-\rho, \rho-r)}^{d-r}(r+j+1-(d-\rho) n)_{+} \\
& -\left\{\left(\begin{array}{c}
d+2 \\
2
\end{array}\right)-\left(\begin{array}{c}
\rho+2 \\
2
\end{array}\right)\right\}_{+} .
\end{aligned}
$$

Then for any quasi-crosscut partition $\Delta_{c}$ with $L$ crosscuts and $V_{I}$ interior vertices,

$$
\operatorname{dim} S_{d}^{r, \rho}\left(\Delta_{c}\right)=\left(\begin{array}{c}
d+2 \\
2
\end{array}\right)+L E_{d}^{r, \rho}+\sum_{i=1}^{V_{I}} F^{r, \rho} d\left(l_{i}\right)
$$

7. Dimension Criterion. A closer investigation into the proof of Theorem 1 in Section 4 not only yields a proof of Theorem 3 stated above but also gives a criterion which guarantees that the lower bound is indeed the actual dimension. To facilitate our presentation of this result, we need the following terminologies.

Let $\Omega^{\prime}$ be any closed polygonal subregion of $\Omega$ such that the boundary of $\Omega^{\prime}$ consists of edges of $\Delta$, and let $\Delta^{\prime}$ be the rectilinear grid partition of $\Omega^{\prime}$ induced by $\Delta$. Since each function in $S_{d}^{r, \rho}\left(\Delta^{\prime}, \Omega^{\prime}\right)$ is the restriction of some function in the original space $S_{d}^{r, \rho}(\Delta, \Omega)$, we may consider $S_{d}^{r, \rho}\left(\Delta^{\prime}, \Omega^{\prime}\right)$ as a subspace of $S_{d}^{r, \rho}(\Delta, \Omega)$. In the following $\left(\Delta^{\prime}, \Omega^{\prime}\right)$ will be called a subgrid region of $(\Delta, \Omega)$, and $S_{d}^{r, \rho}\left(\Delta^{\prime}, \Omega^{\prime}\right)$ the corresponding grid subspace of $S_{d}^{r, \rho}(\Delta, \Omega)$. Any interior vertex of $\Delta^{\prime}$ which can be connected by an edge of $\Delta^{\prime}$ to a boundary vertex of $\Delta^{\prime}$ will be called a nearboundary interior vertex of $\Delta^{\prime}$. Suppose that $A_{0}$ is a near-boundary interior vertex of $\Delta^{\prime}$. Then a subgrid region $\left(\tilde{\Delta}^{\prime}, \tilde{\Omega}^{\prime}\right)$ of $\left(\Delta^{\prime}, \Omega^{\prime}\right)$ will be called an $A_{0}$-deleted subgrid region of $\left(\Delta^{\prime}, \Omega^{\prime}\right)$ if the following conditions are satisfied:

(i) $A_{0}$ is a boundary vertex of $\tilde{\Delta}^{\prime}$,

(ii) the number of interior vertices of $\tilde{\Delta}^{\prime}$ is one less than the number of interior vertices of $\Delta^{\prime}$, and

(iii) the complement of $\tilde{\Omega}^{\prime}$ with respect to $\Omega^{\prime}$ is also a polygonal region.

In addition, the subgrid region $\left(\Delta_{1}^{\prime}, \Omega_{1}^{\prime}\right)$ of $\left(\Delta^{\prime}, \Omega^{\prime}\right)$ is called an $A_{0}$-subgrid region if the following conditions are satisfied:

(i) $A_{0}$ is an interior vertex of $\Delta_{1}^{\prime}$,

(ii) $A_{0}$ is the only interior vertex of $\Delta_{1}^{\prime}$, and

(iii) the complement of $\Omega_{1}^{\prime}$ with respect to $\Omega^{\prime}$ is also a polygonal region.

The idea of considering subgrid regions $\left(\tilde{\Delta}^{\prime}, \tilde{\Omega}^{\prime}\right)$ and $\left(\Delta_{1}^{\prime}, \Omega_{1}^{\prime}\right)$ originates from the induction proof in Schumaker [6]. We have the following result.

THEOREM 5. Let $\Delta$ be an arbitrary rectilinear grid partition of a closed polygonal region $\Omega$ with $V_{I}$ interior vertices where $V_{I}>0$, and let $0 \leq r \leq \rho \leq d$. 
Then

$\operatorname{dim} S_{d}^{r, \rho}(\Delta, \Omega)$

$$
\begin{aligned}
& \geq\left(\begin{array}{c}
d+2 \\
2
\end{array}\right)+E_{I}\left[\left(\begin{array}{c}
d-r+1 \\
2
\end{array}\right)-2\left(\begin{array}{c}
\rho-r+1 \\
2
\end{array}\right)+\left(\begin{array}{c}
(2 \rho-r-d+1)_{+} \\
2
\end{array}\right)\right] \\
& \quad-V_{I}\left[\left(\begin{array}{c}
d+2 \\
2
\end{array}\right)-\left(\begin{array}{c}
\rho+2 \\
2
\end{array}\right)\right]+\sum_{i=1}^{V_{I}} N_{i}^{\rho} .
\end{aligned}
$$

Furthermore, equality holds if and only if for every subgrid region $\left(\Delta^{\prime}, \Omega^{\prime}\right)$ of $(\Delta, \Omega)$ with at least one interior vertex and any $A_{0}$-deleted subgrid region $\left(\tilde{\Delta}^{\prime}, \tilde{\Omega}^{\prime}\right)$ and the corresponding $A_{0}$-subgrid region $\left(\Delta_{1}^{\prime}, \Omega_{1}^{\prime}\right)$ of $\left(\Delta^{\prime}, \Omega^{\prime}\right)$, where $A_{0}$ is any nearboundary interior vertex of $\Delta^{\prime}$, the identity

$$
S_{d}^{r, \rho}\left(\Delta^{\prime}, \Omega^{\prime}\right)=S_{d}^{r, \rho}\left(\tilde{\Delta}^{\prime}, \tilde{\Omega}^{\prime}\right)+S_{d}^{r, \rho}\left(\Delta_{1}^{\prime} \Omega_{1}^{\prime}\right)
$$

is satisfied, where the usual sum of two vector spaces is considered.

Of course, (31) is only a reformulation of (30). We remark, however, that when the degree $d$ is relatively large compared to the order of smoothness $r$ (e.g. $d \geq$ $3 r+2$ ), the smoothness conditions in terms of Bézier nets can be "disentangled" so that the two grid subspaces on the right of (32) can be understood, and it is then feasible to apply condition (32) to study the geometry of the grid $\Delta$ in determining the dimension of the superspline subspaces $S_{d}^{r, \rho}(\Delta, \Omega)$, and particularly the original spline space $S_{d}^{r}(\Delta, \Omega)=S_{d}^{r, r}(\Delta, \Omega)$.

Department of Mathematics

Texas A \& M University

College Station, Texas 77843-3368

E-mail: e443cc@tamvm 1

1. C. K. ChU I, Multivariate Splines, CBMS-NSF Lecture Series in Applied Math., no. 54, SIAM, Philadelphia, PA, 1988.

2. C. K. ChUI \& M. LAI, "On multivariate vertex splines and applications," in Topics in Multivariate Approximation (C. K. Chui, L. L. Schumaker, and F. Utreras, eds.), Academic Press, New York, 1987, pp. 19-36.

3. C. K. Chui \& R. H. WANG, "Multivariate spline spaces," J. Math. Anal. Appl., v. 94, 1983, pp. 197-221.

4. R. H. J. Gmelig Meyling \& P. R. Pfluger, "On the dimension of the spline space $S_{2}^{1}(\Delta)$ in special cases," in Multivariate Approximation Theory III (W. Schempp and K. Zeller, eds.), Birkhäuser, Basel, 1985, pp. 180-190.

5. J. MORGAN \& R. SCOTT, "A nodal basis for $C^{1}$ piecewise polynomials in two variables," Math. Comp., v. 29, 1975, pp. 736-740.

6. L. L. SCHUMAKER, "On the dimension of spaces of piecewise polynomials in two variables," in Multivatiate Approximation Theory (W. Schempp and K. Zeller, eds.), Birkhäuser, Basel, 1979, pp. 396-412.

7. L. L. SCHUMAKER, "Bounds on the dimension of spaces of multivariate piecewise polynomials," Rocky Mountain J. Math., v. 14, 1984, pp. 251-264.

8. L. L. SChUMAKER, "On super splines and finite elements," SIAM J. Numer. Anal. (To appear.)

9. A. ŽENIŠEK, "Polynomial approximation on tetrahedrons in the finite element method," $J$. Approx. Theory, v. 7, 1973, pp. 334-351.

10. A. ŽEnišEK, "A general theorem on triangular $c^{m}$ elements," RAIRO Anal. Numér., v. 22, 1974, pp. 119-127. 